\title{
k-Space Interference of Coherent TDS Electrons for Mean Atomic Displacement Measurements
}

\author{
Rodney A. Herring \\ CAMTEC, MENG, University of Victoria, British Columbia V8N 2Y2 Canada
}

Methods of controlled electron interference in k-space on the diffraction plane by means of an electron biprism have shown that self-interfered diffusely scattered electrons, elastically scattered and inelastically scattered, found outside and in between the Bragg diffracted beams have sufficient coherence to form fringes [1,2]. The diffuse intensity in between the Bragg Diffracted Beams is the thermal diffuse scattering (TDS) of electrons resulting from the lattice vibrations of the atoms in their atomic columns. This abstract reports the formation of fringes within the TDS intensity using diffracted beam interferometry (DBI) to measure the TDS phase that enabled the determination of a mean displacement of the atoms, $u \sim 12 \mathrm{pm}$, in Aluminum in the direction perpendicular to the 111 atomic planes.

Previous DBI experiments have revealed that practically all electron intensities on the diffraction plane originating from all materials, i.e., crystals and amorphous materials, generated from a coherent electron source of a TEM have sufficient coherence to form fringes when self-interfered under a wide range of electron diffraction conditions, which prompted the investigation of the existence of fringes in the TDS intensity. A significant advantage of measuring in k-space versus the image plane is the angular separation of the diffusely scattered electrons essentially acting as a spatial filter.

By measuring the virtual electron source separation, $\Delta S$, using DBI, the phase of the TDS intensity, $k\left(r_{1}-r_{2}\right)$, was measured from the thickness of fringes at a displacement, $y$, from the center of interference of the two Bragg diffracted beams having a path length difference, $r_{1}-r_{2}$ (Fig 1), such that

$$
r_{1}-r_{2}=\Delta S^{\prime} \frac{y}{L}
$$

where $L$ is the camera length. The phase can then be related to the mean square displacement of the atom, $u$, in the angular direction, $\theta_{\mathrm{B}}$, perpendicular to the crystal's Bragg diffracting planes such that

$$
k\left(r_{1}-r_{2}\right)=-16 \pi^{2} u^{2} \sin ^{2} \theta_{B} / \lambda^{2}
$$

where $\lambda$ is the wavelength. Phase measurements (Fig 2) resulted in $u \sim 12 \mathrm{pm}$, a reasonable $2.7 \%$ deviation from the Al lattice parameter, in agreement with the theoretical estimate of $12 \mathrm{pm} \mathrm{[3]} \mathrm{but} \mathrm{far} \mathrm{from} \mathrm{the} \mathrm{recent} \mathrm{experimental} \mathrm{measurements} \mathrm{of}$ $68 \mathrm{pm}$ to $75 \mathrm{pm}$ made on the image plane from lattice images [4]. This is the first time that the TDS's phase has been measured by any means useful for the study of lattice dynamics in solids.

References:

[1] R.A. Herring, "Electron beam coherence measurements using diffracted beam interferometry/holography" JEM 58 (2009) 213.

[2] R.A. Herring, "Coherence of k-space electrons - application to TDS electrons by DBI" Microscopy JEM-2012-00116.R1 (accepted - in print). 
[3] G Radi "Complex Lattice Potentials in Electron Diffraction Calculated for a Number of Crystals" Acta Cryst. A26 (1970) 41.

[4] B. Barton et al., "Atomic Resolution Phase Contrast Imaging and In-Line Holography Using Variable Voltage and Dose Rate" Microscopy and Microanalysis 18 (2012) 982.

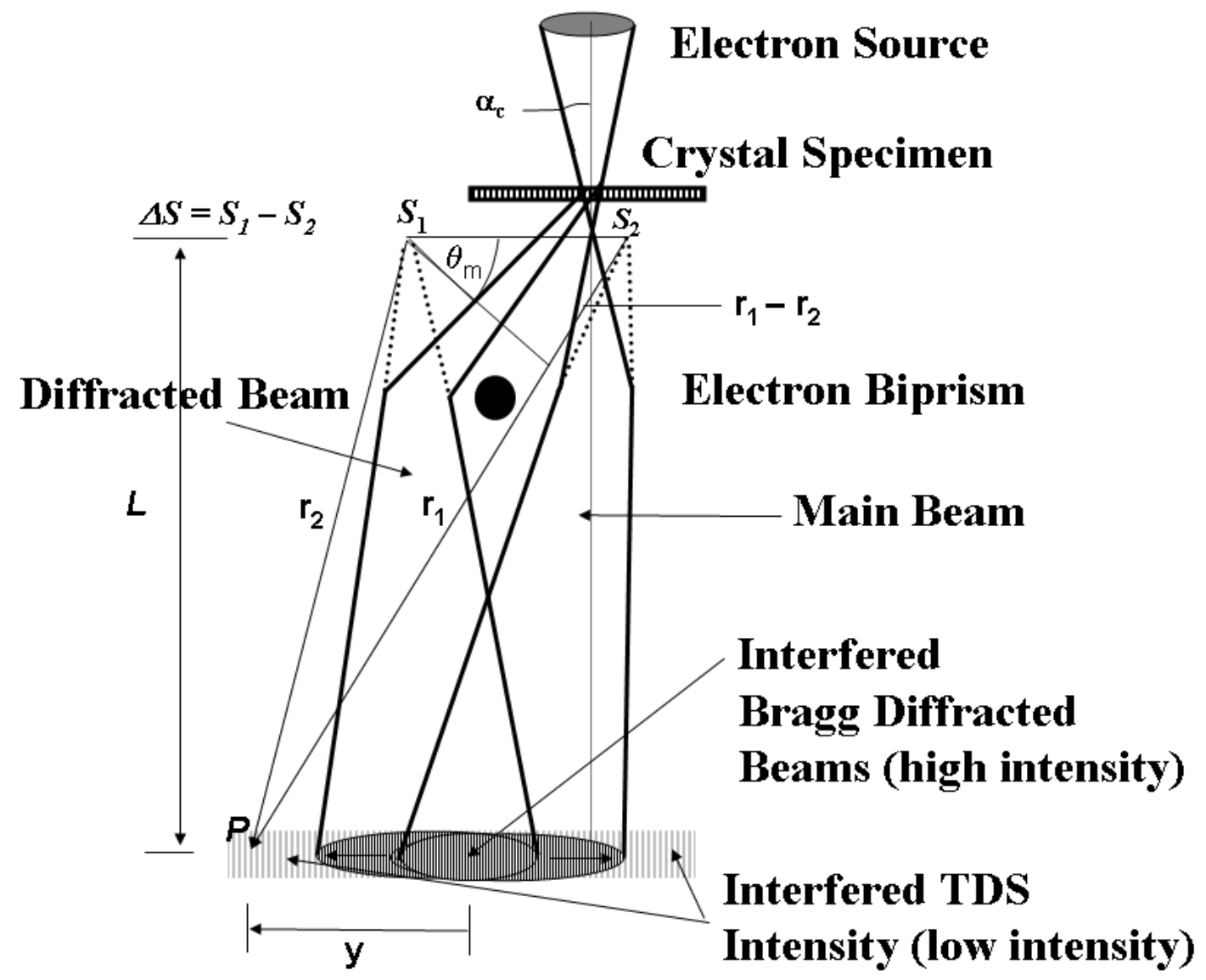

Fig 1 - Schematic showing the virtual sources, $S_{1}$ and $\mathrm{S}_{2}$, path length vectors, $r_{1}$ and $r_{2}$, and their difference, $r_{1}-r_{2}$, and the distance $y$ from the interference center to point $P$.
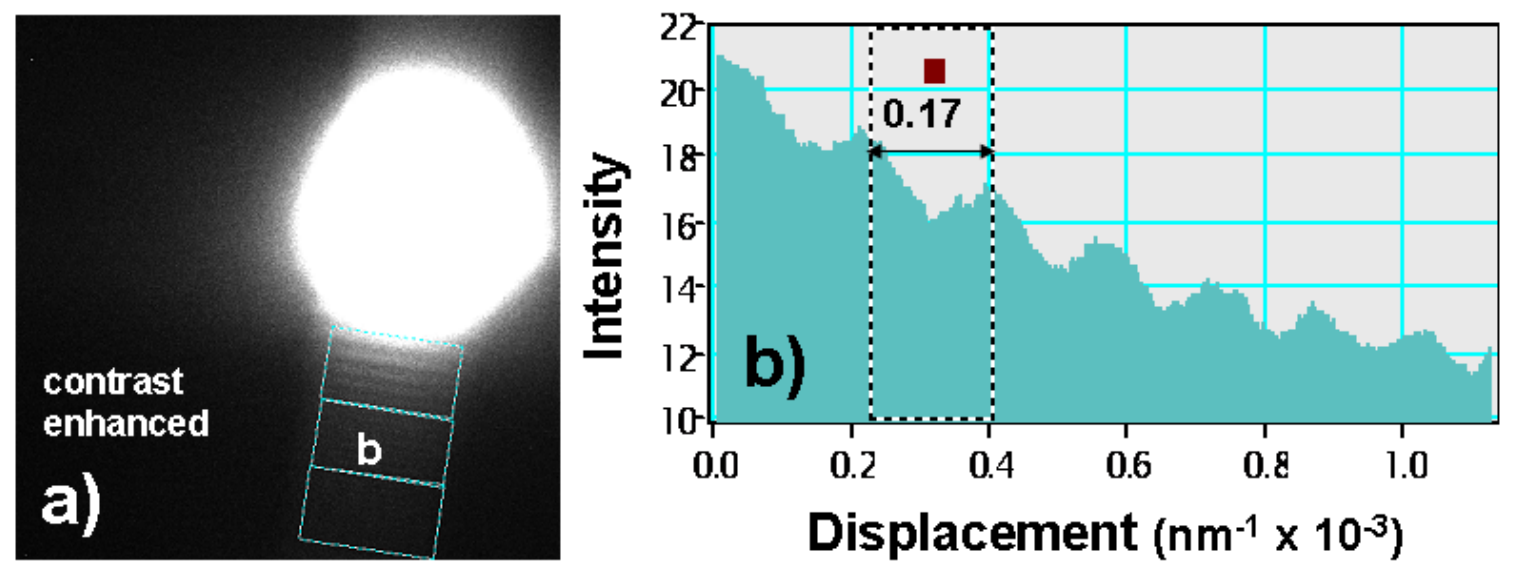

Fig 2 - a) Interference of the 000 and 222 beams of $\mathrm{Al}$ resulting in the formation of fringes in the low intensity in region $b$ shown in $b$ ) used to measure the mean displacement of atoms, $u$. 